\title{
Tracking Single Cells in Live Animals Using a Photoconvertible Near-Infrared Cell Membrane Label
}

\author{
Alicia L. Carlson ${ }^{19}$, Joji Fujisaki ${ }^{19}$, Juwell $\mathrm{Wu}^{1}$, Judith M. Runnels ${ }^{1}$, Raphaël Turcotte ${ }^{1,2}$, \\ Cristina Lo Celso ${ }^{3 \alpha}$, David T. Scadden ${ }^{3,4,5,6}$, Terry B. Strom ${ }^{7}$, Charles P. Lin ${ }^{1,5 *}$
}

1 Center for Systems Biology and Wellman Center for Photomedicine, Massachusetts General Hospital and Harvard Medical School, Boston, Massachusetts, United States of America, 2 Department of Biomedical Engineering, Boston University, Boston, Massachusetts, United States of America, $\mathbf{3}$ Center for Regenerative Medicine, Massachusetts General Hospital, Boston, Massachusetts, United States of America, 4 Cancer Center, Massachusetts General Hospital, Boston, Massachusetts, United States of America, $\mathbf{5}$ Harvard Stem Cell Institute, Cambridge, Massachusetts, United States of America, $\mathbf{6}$ Department of Stem Cell and Regenerative Biology, Harvard University, Cambridge, Massachusetts, United States of America, 7 Transplant Institute, Beth Israel Deaconess Medical Center and Harvard Medical School, Boston, Massachusetts, United States of America

\begin{abstract}
We describe a novel photoconversion technique to track individual cells in vivo using a commercial lipophilic membrane dye, DiR. We show that DiR exhibits a permanent fluorescence emission shift (photoconversion) after light exposure and does not reacquire the original color over time. Ratiometric imaging can be used to distinguish photoconverted from nonconverted cells with high sensitivity. Combining the use of this photoconvertible dye with intravital microscopy, we tracked the division of individual hematopoietic stem/progenitor cells within the calvarium bone marrow of live mice. We also studied the peripheral differentiation of individual T cells by tracking the gain or loss of FoxP3-GFP expression, a marker of the immune suppressive function of $\mathrm{CD}^{+} \mathrm{T}$ cells. With the near-infrared photoconvertible membrane dye, the entire visible spectral range is available for simultaneous use with other fluorescent proteins to monitor gene expression or to trace cell lineage commitment in vivo with high spatial and temporal resolution.
\end{abstract}

Citation: Carlson AL, Fujisaki J, Wu J, Runnels JM, Turcotte R, et al. (2013) Tracking Single Cells in Live Animals Using a Photoconvertible Near-Infrared Cell Membrane Label. PLoS ONE 8(8): e69257. doi:10.1371/journal.pone.0069257

Editor: Marc Tjwa, University of Frankfurt - University Hospital Frankfurt, Germany

Received August 31, 2012; Accepted June 13, 2013; Published August 26, 2013

Copyright: (c) 2013 Carlson et al. This is an open-access article distributed under the terms of the Creative Commons Attribution License, which permits unrestricted use, distribution, and reproduction in any medium, provided the original author and source are credited.

Funding: This work was supported by National Institutes of Health (NIH) grants HL097748, HL97794, CA086355, and HL100402. TBS was supported by NIH grant 5 PO1 Al041521-12, Juvenile Diabetes Research Foundation grants 7-2005-1329 and 4-2007-1057, and the JDRF Center in Immunologic Tolerance. The funders had no role in study design, data collection and analysis, decision to publish, or preparation of the manuscript.

Competing Interests: The authors declare that no competing interests exist.

* E-mail: charles_lin@hms.harvard.edu

a Current address: Division of Cell and Molecular Biology, Imperial College London, London, United Kingdom

9 These authors contributed equally to this work.

\section{Introduction}

Understanding of biological processes would be enhanced and cell-based therapies improved by knowing the exact in vivo location and environmental factors that regulate cell division and differentiation. However, conventional population-based tracking techniques have left many critical questions unresolved. For example, it has been difficult to determine where individual stem cells actually divide and differentiate in vivo, despite extensive research focused on identifying the microenvironment that regulates stem cell fate [1-4]. In conventional studies of cell lineage and development, heterogeneities and possible contaminants in the population of interest have led to ambiguities in the interpretation of the results [5-9].

It is therefore necessary to develop a method to track single-cell division and differentiation in vivo, which will uncover cellular dynamics that are lost in the ensemble averaging of traditional population-based studies. Elegant in vitro demonstrations of stem cell lineage commitment by time-lapse imaging have been described [10,11] and studies of cell movement and cell-cell interactions in live animals have become possible with the development of confocal and multiphoton intravital microscopy
(IVM) [12-16]. In vivo studies of cell division and differentiation, however, are limited by the length of time the animal can be kept under anesthesia (hours). Alternatively, images can be acquired over multiple imaging sessions, provided that a method exists to locate the same field of view when the animal is repositioned on the stage [3,17]. This approach can greatly extend the recording time span, but the missing time gaps between imaging sessions can translate into knowledge gaps (e.g., the target cell can move out of the field of view or other cells can move in and be mistaken as the original cell or its progeny) unless additional measures are taken to mark the cells of interest to ensure that the same cells are being tracked with no mistaken identity.

One way to highlight the cells of interest in vivo for subsequent tracking is to use photoswitchable [18-28] or photoconvertible [18,29-40] fluorescent proteins. However, fluorescent proteins have distinct disadvantages that limit their use for tracking cell division in vivo over long time periods. Firstly, loading fluorescent proteins into cells requires transfection, which can change the phenotype of some target cells. Secondly, after photoconversion, new fluorescent proteins produced by the cell will express the original color. Therefore, significant loss of the photoconverted signal will occur through protein turnover and the photoconverted 
cells revert back to their original color within 24 hours of photoconversion [37,41]. Finally, although transgenic mice expressing photoconvertible fluorescent proteins exist [36,37], in order to visualize cell differentiation, new transgenic mice in which the target cells express the photoconvertible fluorescent proteins together with a second fluorescent proteins that marks the differentiation status or function of the cell will have to be generated.

We have, instead, developed a simple photoconversion technique for long-term tracking of single-cell division and differentiation in vivo using a commercial lipophilic membrane dye, DiR $\left(\mathrm{DiIC}_{18}(7)\right.$; Invitrogen, Carlsbad, CA). DiR can be used to label cells, including freshly isolated cells, with no known effect on their homing or proliferation [3]. The dye exhibits a permanent change in the fluorescence emission spectrum after photoconversion, and ratiometric imaging can be used to distinguish photoconverted and non-photoconverted cells with high sensitivity. The ratio for each cell remains stable with no reversion to the original color. A schematic drawing depicting the concept of cell tracking by photoconversion is shown in Fig. 1. In this drawing, one DiRlabeled cell seen at an initial time point (Fig. 1A) cannot be distinguished among multiple cells at the same location at a later time point (Fig. 1B). Therefore, proliferation of the initial cell of interest (Fig. 1A) cannot be distinguished from new cell infiltration with certainty. Using light activation to induce photoconversion, the fluorescence emission of only the cell of interest can be changed (Fig. 1C), highlighting that cell so that it can be followed longitudinally to track its fate, including both cell division (Fig. 1D) as well differentiation (when marked by a genetically encoded fluorescent reporter) (Fig. 1E). During cell division, the progeny will retain the photoconverted fluorescence color (Fig. 1D). During differentiation, a photoconverted cell will change its fluorescence color when a reporter gene is turned on or off (Fig. 1E).
Using a custom IVM system and the photoconversion technique, we tracked the division of individual hematopoietic stem/progenitor cells (HSPCs) and mature T cells in the calvarium bone marrow (BM) of live mice for up to $135 \mathrm{~h}$. We have further tracked the peripheral differentiation of DiR-labeled $\mathrm{T}$ cells and regulatory $\mathrm{T}$ cells by monitoring the gain or loss of the expression of green fluorescence protein (GFP) co-expressed with FoxP3, a transcription factor that governs the immune suppressive function of $\mathrm{CD}^{+} \mathrm{T}$ cells [42-44]. These data show that optically marking cells by the DiR dye-photoconversion technique enables a single cell or population of cells to be tracked over long time periods in vivo.

\section{Methods}

\section{Ethics statement}

Animal studies were conducted under the approval and guidelines of the Massachusetts General Hospital Subcommittee on Research Animal Care. Animals were maintained in accordance with the "Guide for the Care and Use of Laboratory Animals" (National Research Council, 1996). Massachusetts General Hospital (MGH) is registered with the U.S. Department of Agriculture Animal and Plant Health Inspection Service (Certificate No. 14-R-0014) and the Massachusetts Department of Public Health (License No. 11-0022) as a licensed animal research facility. MGH files an annual Letter of Assurance (File No. A3596-01) with the NIH Office of Laboratory Animal Welfare confirming compliance with PHS regulations pertaining to laboratory animal care and use. In addition, the hospital is accredited by the Association for the Assessment and Accreditation of Laboratory Animal Care International (AAALAC). All procedures were performed on sedated animals under Ketamine/Xylazine anesthesia

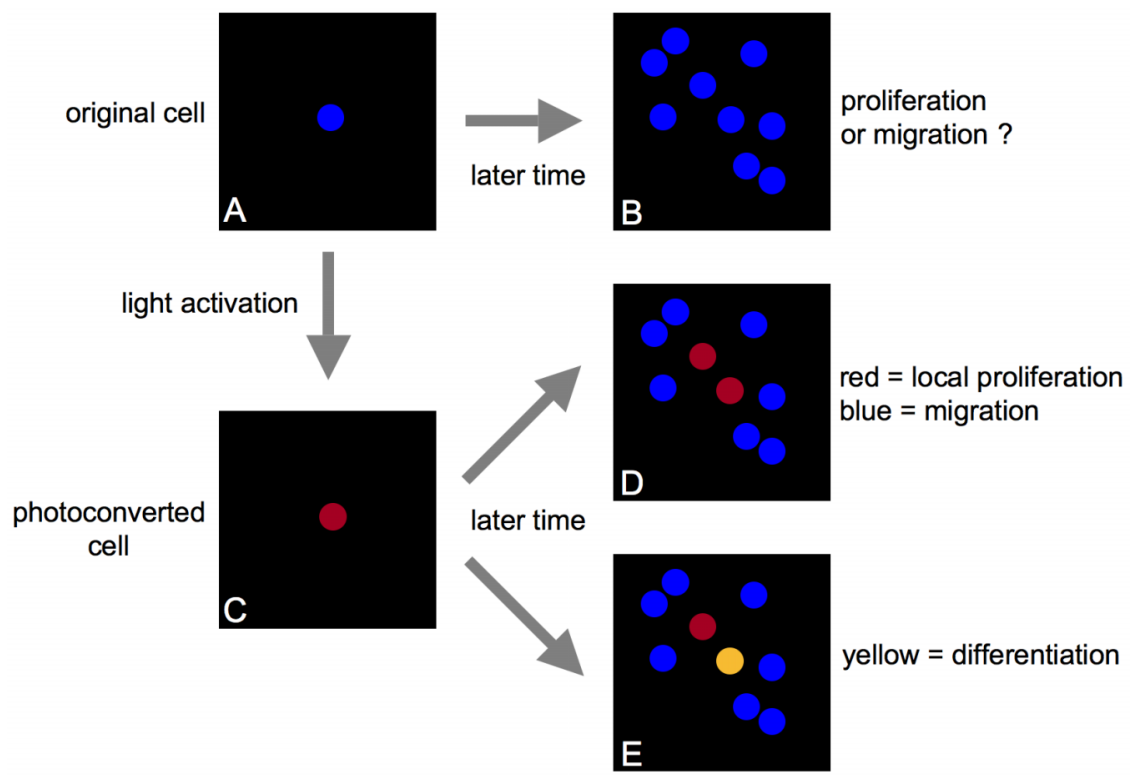

Figure 1. Schematic drawing of in vivo photoconversion method to track fate of a single cell. (A) One DiR-labeled cell (blue circle) in the field of view. (B) Without photoconversion, when the same area is imaged at a later time point, additional DiR-labeled cells may be found in the same area, such that proliferation of the single cell viewed previously cannot be distinguished from new cell infiltration. (C) Photoconversion of the DiRlabeled cell (A, blue circle) changes its fluorescence emission (C, red circle), highlighting that cell so that it can be followed longitudinally to track its fate, including both cell division (D) as well differentiation when utilizing a fluorescent reporter gene to mark cell differentiation or function (E). During cell division, the progeny will retain the photoconverted fluorescence color (single red cell in (C) becomes two red cells in (D) through cell division). During differentiation, a photoconverted cell will change its fluorescence color when a reporter gene is turned on or off (red cell in (E) becomes yellow when GFP reporter is turned on).

doi:10.1371/journal.pone.0069257.g001 
(100 mg/kg Ketamine+15 mg/kg Xylazine), appropriate analgesia was administered as needed, and all efforts were made to minimize suffering.

\section{Animals}

FoxP3 GFP knock-in mice (C57BL/6 background) and C57BL/6 mice were purchased from Jackson Laboratory (Bar Harbor, ME). RAG2 $2^{-1-}$ mice were purchased from Taconic (Hudson, NY).

\section{HSPC isolation}

Whole bone marrow was obtained from C57BL/6 mice by crushing the femur, tibia, iliac, humerus, and vertebral bones. Cells were pooled, washed, and incubated in a lineage cocktail consisting of biotinylated B220, Mac1, GR-1, CD3a, CD8a, CD4 and Ter1 19 antibodies (BD Bioscience, Franklin Lakes, NJ). After washing, cells were incubated with MACS-SA beads (Miltenyi, Invitrogen, Carlsbad, CA) per manufacturer's protocol, washed again, and then separated on an LD depletion column in a MidiMACS separation unit in order to remove lineage-specific cell populations. Lineage-negative cells were then stained with Ckit (eBioscience, San Diego, CA) and Scal antibodies (BD Bioscience, Franklin Lakes, NJ). Subsequently, Scal+Ckit+Lin- cells were isolated using a FACS Aria (Becton Dickinson, Franklin Lakes, NJ).

\section{T cell isolation}

Spleen and peripheral cervical, axillary, and inguinal lymph nodes were removed from C57BL/6 FoxP3-GFP mice and cells were harvested by disruption and aspiration of the tissues. Cells were centrifuged and re-suspended in media prior to ACK lysis of the red blood cells. After staining with anti-CD4 antibody (BD Bioscience, Franklin Lakes, NJ), GD4+GFP- cells or CD4+GFP+ cells were isolated using a FACS Aria (Becton Dickinson, Franklin Lakes, NJ).

\section{DiR cell labeling}

Cells were labeled with the fluorescent, lipophilic carbocyanine $\operatorname{DiIC}_{18}(7) \quad$ (1,1'-dioctadecyl-3,3,3', 3' -tetramethylindotricarbocyanine iodide) ('DiR', Invitrogen, Carlsbad, CA). For in vitro work (Figs. 2 and 3), cells were labeled for $10-20 \mathrm{~min}$ at $37^{\circ} \mathrm{C}$ at a concentration of $5 \mu \mathrm{M}$ in RPMI with $0.1 \% \mathrm{BSA}$. For the in vivo work (Figs. 4, 5, and 6), cells were labeled for $25 \mathrm{~min}$ at $37^{\circ} \mathrm{C}$ at a concentration of $15 \mu \mathrm{M}$ in RPMI with $1 \%$ FBS. Following labeling, cells were washed a minimum of two times to remove unbound dye.

\section{Bone marrow transplantation}

DiR-labeled HSPGs were intravenously injected via the tail vein into 9.5 Gy-irradiated recipient mice.

\section{Fluorescence spectroscopy}

Fluorescence emission spectra of photoconverted and nonphotoconverted DiR-labeled cells were acquired using a SPEX FluoroMax-3 (Horiba Scientific, Edison, NJ) at $632 \mathrm{~nm}$ excitation with a $3 \mathrm{~nm}$ slit width. Emission was collected from $640 \mathrm{~nm}$ to $845 \mathrm{~nm}$, using a $5 \mathrm{~nm}$ slit width. Excitation spectra of the cells were acquired for $780 \mathrm{~nm}$ emission and $670 \mathrm{~nm}$ emission, both with a $3 \mathrm{~nm}$ slit width. Excitation was collected from $400 \mathrm{~nm}$ to $775 \mathrm{~nm}$ and from 400 to $665 \mathrm{~nm}$, respectively, both with a $5 \mathrm{~nm}$ slit width. The intensities of the emission spectra were then corrected to account for differences in the excitation absorption derived from the excitation spectra.
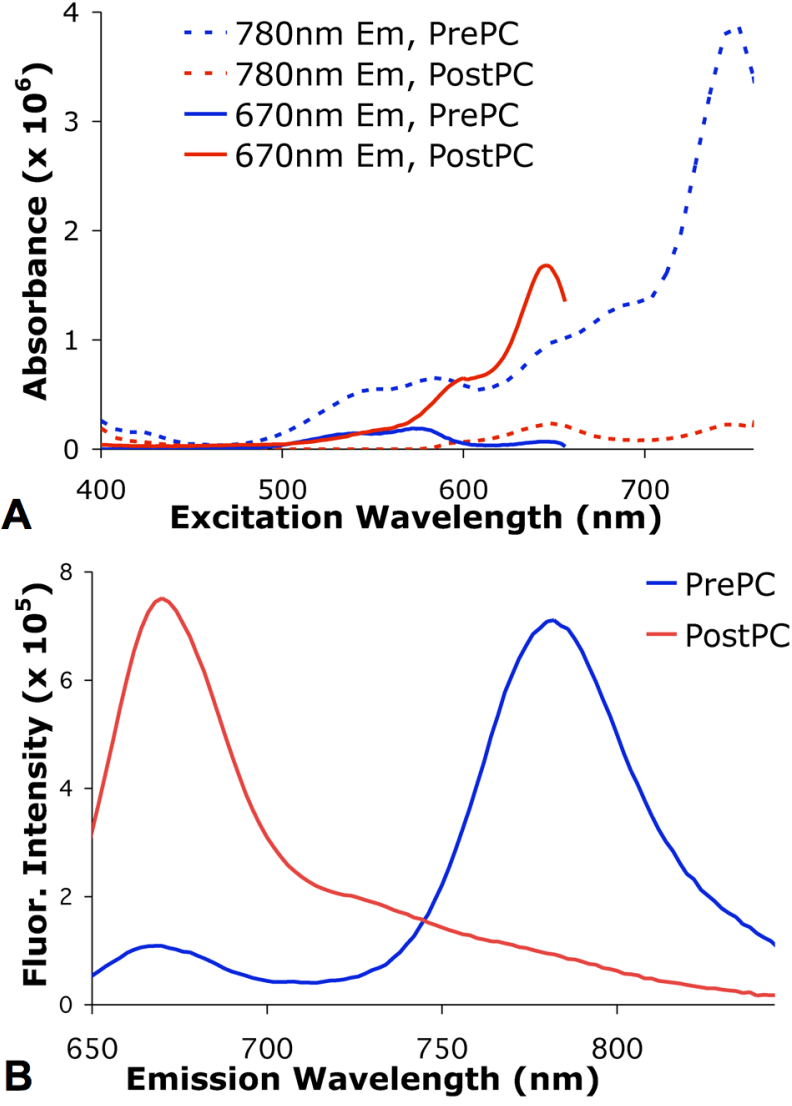

Figure 2. DiR dye spectra before and after photoconversion. (A) Fluorescence excitation spectra of DiR-labeled cells before (PrePC) and after (PostPC) photoconversion acquired at $670 \mathrm{~nm}$ and $780 \mathrm{~nm}$ emission. (B) Fluorescence emission spectra of DiR-labeled cells before (PrePC) and after (PostPC) photoconversion when excited at $632 \mathrm{~nm}$, showing a significant shift in the fluorescence peak from $780 \mathrm{~nm}$ to $670 \mathrm{~nm}$ following photoconversion.

doi:10.1371/journal.pone.0069257.g002

\section{In vivo confocal imaging}

Images were acquired with a non-commercial confocal/multiphoton microscope specifically designed for live animal imaging. The microscope design is described in detail in [45]. Briefly, the system contains three continuous wave lasers, yielding four excitation wavelengths at $491 \mathrm{~nm}$ (Dual Calypso, Cobolt AB, Stockholm, Sweden), $532 \mathrm{~nm}$ (Dual Calypso, Cobolt AB, Stockholm, Sweden), $561 \mathrm{~nm}$ (Jive, Cobolt AB, Stockholm, Sweden), and $635 \mathrm{~nm}$ (Radius, Coherent Inc., Santa Clara, CA) The system also contains a Mai Tai femtosecond pulsed laser (Coherent Inc., Santa Clara, CA) for multi-photon and second-harmonic imaging as well as single-photon photoconversion. The scan engine of the system consists of a spinning polygon (Lincoln Laser, Phoenix, AZ) and galvanometer (Cambridge Technology, Bedford, MA), producing images of $500 \times 500$ pixels at a frame rate of 30 frames per second. The system contains five photomultiplier tube (PMT) detectors, four for confocal imaging and one for two-photon and second-harmonic imaging. High-resolution images were acquired using a $30 \times 0.9$ NA water-immersion objective (Lomo, St. Petersburg, Russia) which yielded a field of view of $660 \times 660 \mu \mathrm{m}$.

Ex vivo images were acquired of cells on glass cover slips, and in vivo images were acquired of transplanted cells in the skull BM in live mice. Mice were anaesthetized by intraperitoneal injection of a Ketamine/Xylazine cocktail (100 mg/kg Ketamine+15 mg/kg 


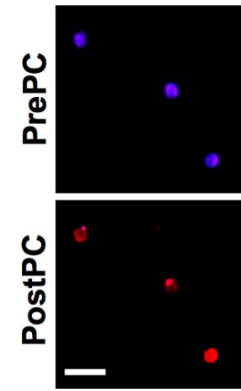

A

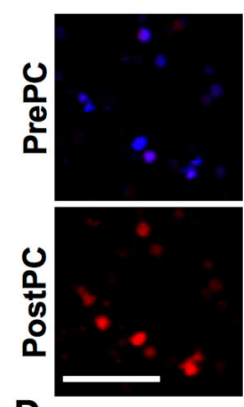

D

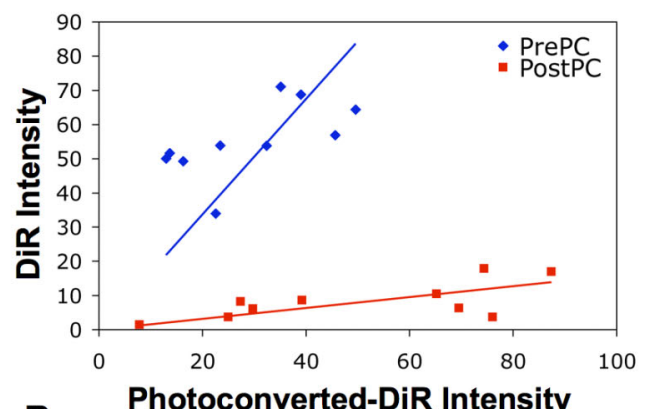

B

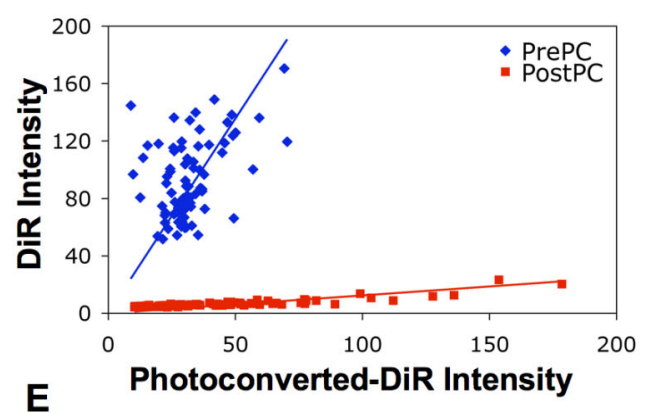

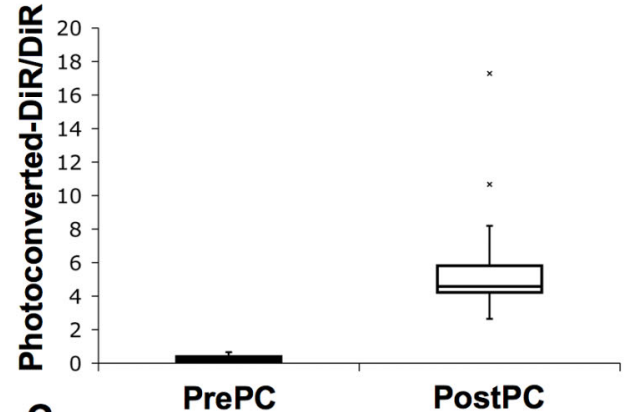

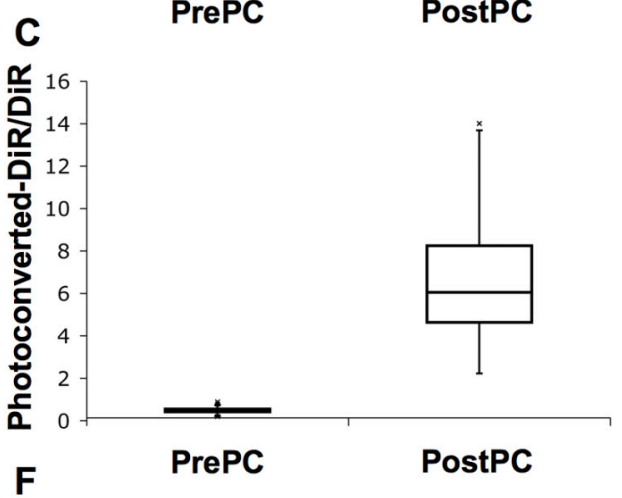

Figure 3. Ex vivo photoconversion of DiR-labeled cells. (A) Fluorescence confocal images of ex vivo DiR-labeled HSPCs acquired before (PrePC) and after (PostPC) photoconversion (blue: DiR, 760-810 nm; red: photoconverted-DiR, 660-760 nm). Scale bar: $50 \mu \mathrm{m}$. (B) Plot of fluorescence intensity of ex vivo HSPCs before (PrePC) and after (PostPC) photoconversion for each individual cell. (C) Boxplot of the ratios of the photoconvertedDiR intensity to the DiR intensity, showing ability to photoconvert DiR-labeled stem and progenitor cells and to distinguish the change in the fluorescence intensity ratio after photoconversion $\left(p=8.36 \times 10^{-4}\right)$. (D) Fluorescence confocal images of ex vivo DiR-labeled T cells acquired before (PrePC) and after (PostPC) photoconversion (blue: DiR, $>770 \mathrm{~nm}$; red: photoconverted-DiR, $670-720 \mathrm{~nm}$ ). Scale bar: $50 \mu \mathrm{m}$. (E) Plot of fluorescence intensity of ex vivo T cells before (PrePC) and after (PostPC) photoconversion. (F) Boxplot of the fluorescence intensity ratios, also showing ability to photoconvert DiR-labeled T cells and to distinguish the change in fluorescence after photoconversion $\left(p=1.15 \times 10^{-33}\right)$. doi:10.1371/journal.pone.0069257.g003

Xylazine) and prepared for in vivo imaging by making a small incision in the scalp, creating a skin flap to expose the underlying dorsal surface of the skull. Mice were held in a temperaturecontrolled tube, positioned on the microscope stage. Highresolution images of cellular details were obtained through the intact mouse skull at depths up to $150 \mu \mathrm{m}$ from the dorsal surface of the skull. For imaging, DiR was excited using the $635 \mathrm{~nm}$ helium-neon laser (Radius). The emitted fluorescence was detected in two channels, either through a $710 \pm 50 \mathrm{~nm}$ bandpass filter (HHQ710/100M, Chroma Technologies, Rockingham, VT) and $785 \pm 25 \mathrm{~nm}$ bandpass filter (OS02770, Chroma Technologies, Rockingham, VT) (set-up A, Figs. $3 \mathrm{~A}$ and $3 \mathrm{~B}$ ) or through a $695 \pm 27.5 \mathrm{~nm}$ bandpass filter (XF3076 695AF55, Omega Optical, Brattleboro, VT) and $770 \mathrm{~nm}$ longpass filter (HQ770LP, Chroma Technologies, Rockingham, VT) (set-up B, Figs. 3C, 3D, 4, 5, and 6). GFP was excited with the $491 \mathrm{~nm}$ solid-state laser (Dual Calypso) and detected through a $528 \pm 19 \mathrm{~nm}$ bandpass filter (FF01-528/38-25, Semrock, Rochester, NY). Images were captured, after averaging thirty frames, using a Macintosh computer equipped with an Active Silicon snapper card (Active Silicon Chelmsford, MA). Each channel was acquired individually, but simultaneously, in 8-bit grayscale and merged to form an RGB image, using custom developed software (iPhoton). Multiple time points were taken between 1 and 135 hours after cell injection. At the completion of the initial imaging session, the skull was flushed with sterile saline, and the scalp skin flap was closed using 5-0 nonabsorbable nylon sutures. For subsequent imaging sessions, the sutures were removed and the area flushed and swabbed with sterile saline.

\section{Photoconversion}

Injected cells were located based on their DiR fluorescence emission. Fluorescence images were acquired before and after photoconversion of the dye for ratiometric imaging. In vitro photoconversion was accomplished by illuminating the cell(s) of interest with $8-15 \mathrm{~mW}$ of power (scanned over $660 \times 660 \mu \mathrm{m}$ ) at $750 \mathrm{~nm}$ (Mai Tai, SpectraPhysics, Newport, Santa Clara, CA) for 5-10 s. In vivo photoconversion was accomplished with $32-45 \mathrm{~mW}$ of power scanned over $660 \times 660 \mu \mathrm{m}$ for $20 \mathrm{~s}$. To photoconvert a single cell, a physical mask was placed at an intermediate image plane to restrict the exposure area to the size of a single cell. The ratio of the $685-735 \mathrm{~nm}$ (red) fluorescence (imaging set-up A, Figs. 3A and 3B) or 668-722 $\mathrm{nm}$ (red) fluorescence (imaging set-up $B$, Figs. 3C, 3D, 4, 5, and 6) to the fluorescence greater than $770 \mathrm{~nm}$ (near-infrared, NIR) before and after photoconversion was then used to distinguish between photoconverted and nonphotoconverted cells within an area of tissue.

\section{Data analysis}

Data analysis was conducted on a cell-by-cell basis. The average intensity of each cell within the blue and the red channels of the image was determined using either ImageJ (NIH, Bethesda, MD) or Matlab (Mathworks, Natick, MA). For the ex vivo images and the $1 \mathrm{~h}$ time point in vivo images, the same region of the images acquired before and after photoconversion was analyzed. The ratio of the intensity in the red channel (685-735 $\mathrm{nm}$ for imaging set-up $A$ or $668-722 \mathrm{~nm}$ for imaging set-up B) to the intensity in the blue channel $(>770 \mathrm{~nm}$ ) was calculated for each cell. A two-tailed, two-sample paired t-test with an alpha value of 0.001 was applied 


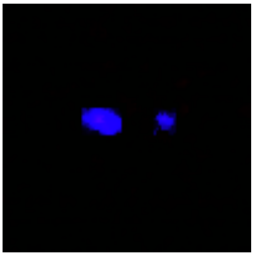

A

PrePC

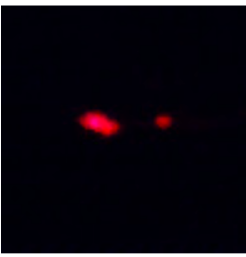

PostPC

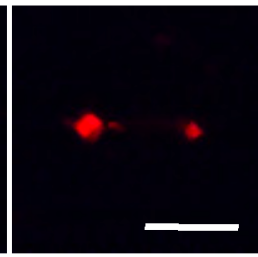

48h PostPC

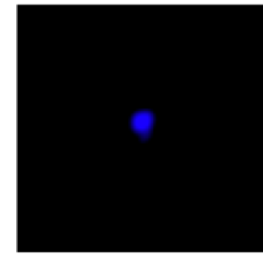

PrePC

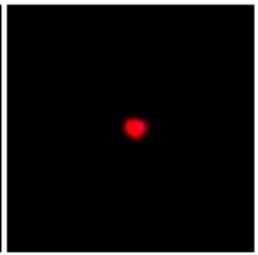

PostPc

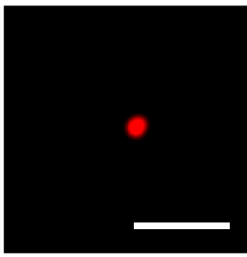

48h PostPC

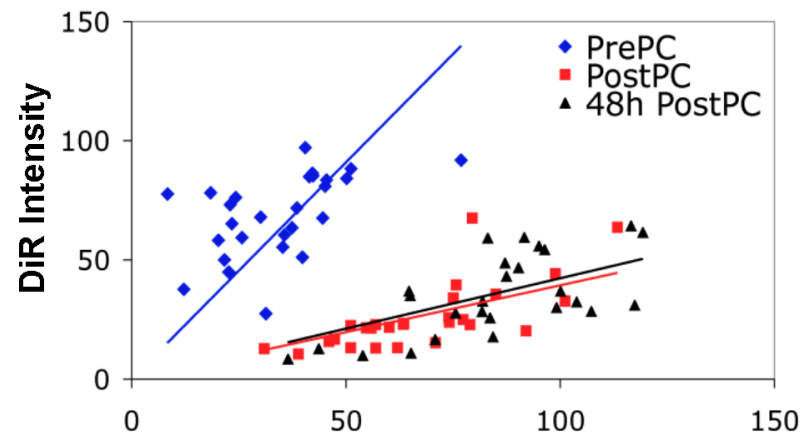

$\mathbf{B}$

Photoconverted-DiR Intensity

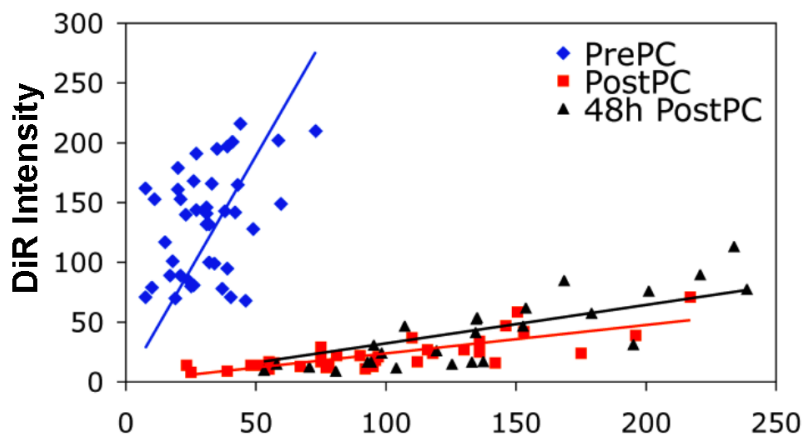

E

Photoconverted-DiR Intensity
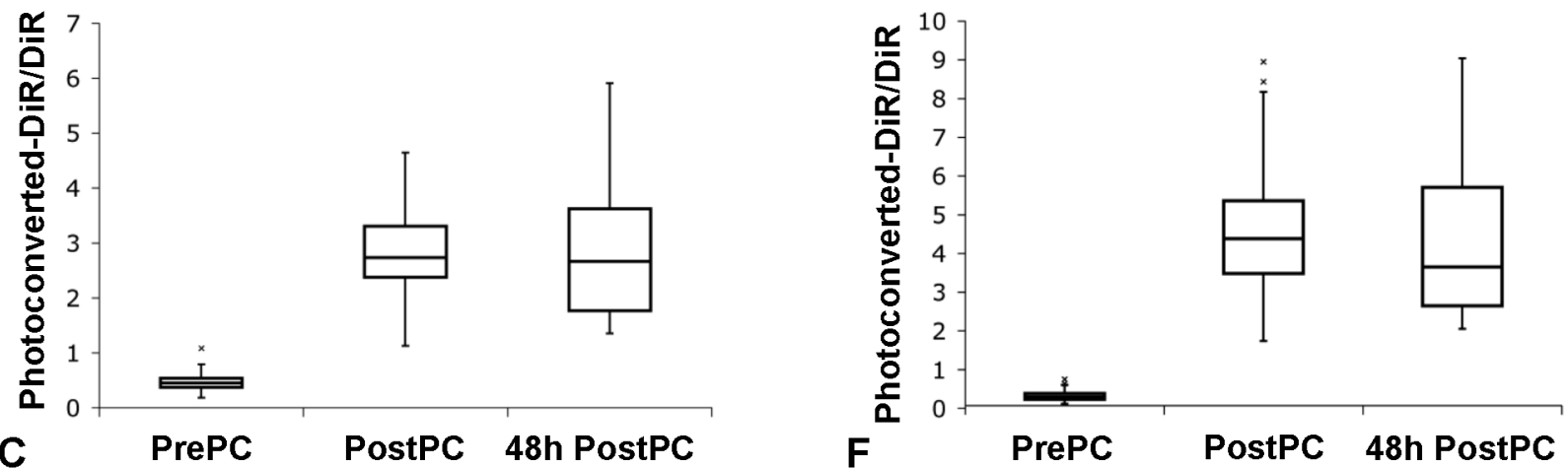

Figure 4. In vivo photoconversion of DiR-labeled cells. (A) In vivo fluorescence confocal images of DiR-labeled HSPCs acquired before (PrePC), immediately after (PostPC), and $48 \mathrm{~h}$ after ( $48 \mathrm{~h}$ PostPC) photoconversion within the skull BM of a live mouse (blue: DiR, $>770 \mathrm{~nm}$; red: photoconverted-DiR, 670-720 nm). Scale bar: $50 \mu \mathrm{m}$. (B) Plot of fluorescence intensity of in vivo HSPCs before (PrePC), immediately after (PostPC), and $48 \mathrm{~h}$ after ( $48 \mathrm{~h}$ PostPC) photoconversion for each individual cell. (C) Boxplot of the ratios of the photoconverted-DiR intensity to the DiR intensity, showing ability to photoconvert cells within the skull BM of live mice and to distinguish the change in the fluorescence intensity ratio after photoconversion ( $p_{\text {pre-post }}=7.72 \times 10^{-14}$ ) as well as show the stability of the photoconversion in vivo over time $\left(p_{\text {post }-48 \text { hpost }}=0.82\right.$ ). (D) In vivo fluorescence confocal images of DiR-labeled T cells acquired before (PrePC), immediately after (PostPC), and $48 \mathrm{~h}$ after (48 h PostPC) photoconversion within the skull BM of a live mouse (blue: DiR, $>770 \mathrm{~nm}$; red: photoconverted-DiR, $670-720 \mathrm{~nm}$ ). Scale bar: $50 \mu \mathrm{m}$. (E) Plot of fluorescence intensity of in vivo T cells before (PrePC), immediately after (PostPC), and $48 \mathrm{~h}$ after (48 h PostPC) photoconversion. (F) Boxplot of the fluorescence intensity ratios showing ability to distinguish the change in fluorescence after photoconversion $\left(p_{\text {pre-post }}=2.59 \times 10^{-16}\right.$ ) as well as show the stability of the photoconversion in vivo over time ( $p_{\text {post-48hpost }}=0.94$ ).

doi:10.1371/journal.pone.0069257.g004

to all before (PrePG) - after (PostPG) comparisons. A two-tailed, two-sample unpaired t-test, assuming equal variances (based on evaluation by corresponding $\mathrm{f}$ test), with an alpha value of 0.001 was applied to the immediately after (PostPC) - $48 \mathrm{~h}$ after $(48 \mathrm{~h}$ Post PC) comparison. A p-value of less than 0.001 indicated that the samples were statistically different. The boxplots are drawn such that the bottom of the box represents the first quartile (Q1, $25^{\text {th }}$ percentile), the top of the box represents the third quartile (Q3, $75^{\text {th }}$ percentile), the center line represents the median value, and the whiskers extend to the highest and lowest data points or 1.5 times the interquartile range (Q3-Q1), whichever is shorter. Outlier points, those that are greater than 1.5 times the interquartile range, are denoted by x's.

\section{Results}

\section{DiR photoconversion}

Figure 2 shows the excitation and emission spectra of DiRlabeled cells before and after photoconversion. Fluorescence excitation peaks are at $750 \mathrm{~nm}$ prior to photoconversion and $650 \mathrm{~nm}$ after photoconversion. Illumination at $750 \mathrm{~nm}$ is used to induce the photoconversion of the dye. The fluorescence emission spectra, acquired with $632 \mathrm{~nm}$ excitation, are shown in Fig. 2B. After photoconversion, the fluorescence emission peak at $780 \mathrm{~nm}$ decreased while the peak at $670 \mathrm{~nm}$ increased.

Fluorescence confocal images of DiR-labeled HSPCs (Fig. 3A) and $\mathrm{T}$ cells (Fig. 3D) acquired ex vivo with $635 \mathrm{~nm}$ excitation 

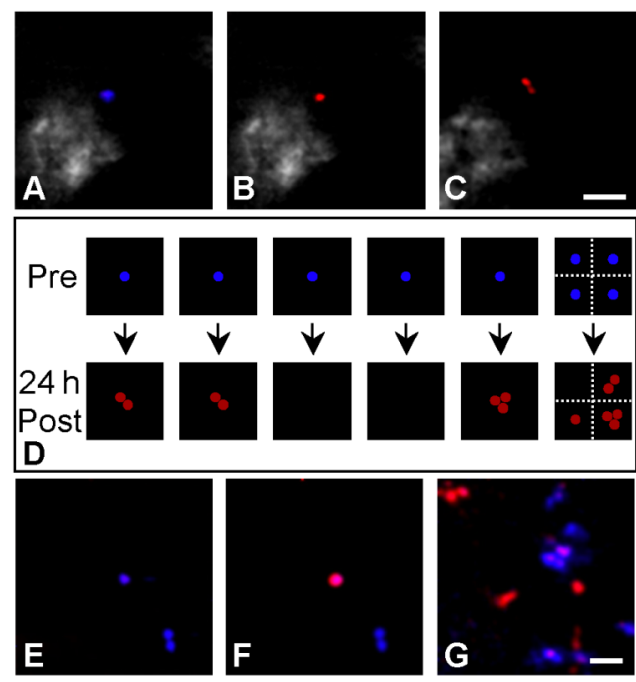

Figure 5. In vivo tracking of hematopoietic stem/progenitor cell proliferation. Fluorescence confocal images of DiR-labeled HSPCS acquired in the skull BM of mice (A) before, (B) immediately after, and (C) $24 \mathrm{~h}$ after in vivo photoconversion (blue: DiR, $>770 \mathrm{~nm}$; red: photoconverted-DiR, $670-720 \mathrm{~nm}$ ). Image (C) shows proliferation of the photoconverted cell. The drawing in figure (D) represents the results of tracking nine cells $24 \mathrm{~h}$ after photoconversion in six mice; each square represents one mouse. Series $(E)-(G)$ demonstrates the ability to track HSPCs over long, discontinuous time periods by showing images acquired within the skull bone marrow (E) before, (F) immediately after, and (G) $135 \mathrm{~h}$ after in vivo photoconversion (blue: DiR, $>770 \mathrm{~nm}$; red: photoconverted-DiR, $670-720 \mathrm{~nm}$ ). Scale bars: $50 \mu \mathrm{m}$.

doi:10.1371/journal.pone.0069257.g005

immediately before and after photoconversion show a characteristic change in the fluorescence emission from near-infrared (NIR) (blue channel) to red (red channel) wavelengths following photoconversion, corresponding to the spectral change seen in Fig. 2B. Ratiometric analysis of the images can be used to distinguish photoconverted cells from non-photoconverted cells; non-photoconverted cells will have fluorescence ratio $R_{b}$ (ratio before photoconversion) while photoconverted cells (and their daughter cells) will have fluorescence ratio $R_{a}$ (ratio after photoconversion). A scatter plot of the average fluorescence intensity of each cell in both the blue and red channels of the image, analyzed before and after photoconversion within a single ex vivo experiment per cell type, is plotted in Figs. 3B and 3E. From this data, the fluorescence ratio of the intensities in the two channels was calculated for each cell (Figs. 3C and 3F). The average fluorescence ratio of the HSPCs after photoconversion was 12.1 times higher than the ratio prior to photoconversion $\left(\mathrm{R}_{\mathrm{b}}=0.53 \pm 0.21, \mathrm{R}_{\mathrm{a}}=6.42 \pm 4.24, \mathrm{p}=8.36 \times 10^{-4}\right)$. Similarly, the average fluorescence ratio of the $\mathrm{T}$ cells after photoconversion was 17.6 times higher than the ratio prior to conversion $\left(\mathrm{R}_{\mathrm{b}}=0.36 \pm 0.12, \quad \mathrm{R}_{\mathrm{a}}=6.35 \pm 2.54, \mathrm{p}=1.15 \times 10^{-33}\right)$, suggesting that good distinction between photoconverted and non-photoconverted cells can be achieved.

\section{In vivo photoconversion of DiR-labeled cells}

The ability to distinguish between photoconverted and nonphotoconverted cells in vivo was then determined. Lethally irradiated C57BL/6 mice were injected with DiR-labeled HSPCs and imaged using IVM one hour after injection. HSPCs were located within the skull BM of live mice based on their DiR fluorescence before photoconversion and were imaged before and after photoconversion as well as $48 \mathrm{~h}$ after photoconversion
(Fig. 4A). The average fluorescence intensity of each cell, from two in vivo experiments per cell type, in both the blue and red channels of the image, is shown in Fig. 4B. After in vivo photoconversion, cells yielded an average red-to-NIR fluorescence (red-to-blue channel intensity) ratio 5.7 times higher than the ratio prior to photoconversion (Fig. $4 \mathrm{C}, \mathrm{R}_{\mathrm{b}}=0.52 \pm 019, \mathrm{R}_{\mathrm{a}}=2.97 \pm 0.88$, $\left.\mathrm{p}=7.72 \times 10^{-14}\right)$. Figures $4 \mathrm{~B}$ and $4 \mathrm{C}$ also show that the fluorescence ratio is stable $48 \mathrm{~h}$ after photoconversion $\left(R_{a}=2.97 \pm 0.88\right.$ immediately after and $2.91 \pm 1.2548 \mathrm{~h}$ after photoconversion, $\mathrm{p}=0.82$ ).

The same ratiometric analysis was repeated for images of injected T cells acquired in vivo in the mouse skull BM. Figure 4D shows in vivo images of $\mathrm{T}$ cells before, immediately after, and 48 hours after photoconversion. Figures $4 \mathrm{E}$ and $4 \mathrm{~F}$ show a similar change in the average fluorescence ratio before and after photoconversion $\quad\left(\mathrm{R}_{\mathrm{b}}=0.25 \pm 0.13, \quad \mathrm{R}_{\mathrm{a}}=4.58 \pm 1.68\right.$, $\left.\mathrm{p}=2.59 \times 10^{-16}\right)$. They also show that the fluorescence ratio is stable $48 \mathrm{~h}$ after photoconversion $\left(\mathrm{R}_{\mathrm{a}}=4.58 \pm 1.68\right.$ immediately after and $4.54 \pm 2.30 \quad 48 \mathrm{~h}$ after photoconversion, $\mathrm{p}=0.94$ ), supporting the use of the dye-photoconversion method to track the fate of DiR-labeled cells over extended time periods.

\section{In vivo photoconversion of DiR-labeled HSPCs to track proliferation}

Photoconverted cells were then tracked longitudinally, using the photoconversion method and calculated fluorescence ratio to track individual cell division within the $\mathrm{BM}$ of live mice. Lethally irradiated C57BL/6 mice were injected with DiR-labeled HSPCs; single cells were targeted and photoconverted one hour after injection. These cells were then imaged again $24 \mathrm{~h}$ after photoconversion. In five of the six recipient mice, only one cell within the entire skull BM was photoconverted, to ensure that any photoconverted cells detected at a later time point were progeny from this single converted cell, while in the remaining recipient, four cells were photoconverted at well-separated locations within the BM giving a total of nine cells at the start of the series of experiments. Figures $5 \mathrm{~A}-5 \mathrm{C}$ show an example of one of the cells before, immediately after, and $24 \mathrm{~h}$ after photoconversion, respectively. Of note, the doublet seen at $24 \mathrm{~h}$ (Fig. 5C) must have come from division of the original cell, because only one cell in the entire mouse was photoconverted. Figure 5D shows the outcome of the follow-up imaging of all nine cells at $24 \mathrm{~h}$ after photoconversion (each square box denotes a single mouse). From six of the nine photoconverted cells, we observed one singlet, three doublets, and two triplets at the same location in the BM $24 \mathrm{~h}$ later, indicating that HSPCs proliferate at the location where they initially home within one hour after intravenous injection.

Interestingly, in the remaining three cases, the photoconverted cell could not be found in the original location or in other regions of the skull BM. It is not likely that the photoconverted HSPCs underwent so many cell divisions within $24 \mathrm{~h}$ that the dye was diluted below detection, as we can track cells after at least three cell divisions (see Fig. 6C discussed below). A more plausible explanation is that the HSPCs either migrated deeper into the $\mathrm{BM}$, beyond our $150 \mu \mathrm{m}$ imaging depth, or migrated out of the skull, possibly by re-entering the circulation. We cannot, however, rule out the possibility that some of the injected cells lost viability and were removed by host cells.

We were also able to perform long-term imaging of HSPCs within the skull bone marrow by tracking photoconverted cells for $135 \mathrm{~h}$ (5.6 days). Figures 5E-5G show an example of one cell before, immediately after, and $135 \mathrm{~h}$ after photoconversion, respectively, again supporting the use of the dye-photoconversion to track the fate of DiR-labeled cells over extended time periods. 

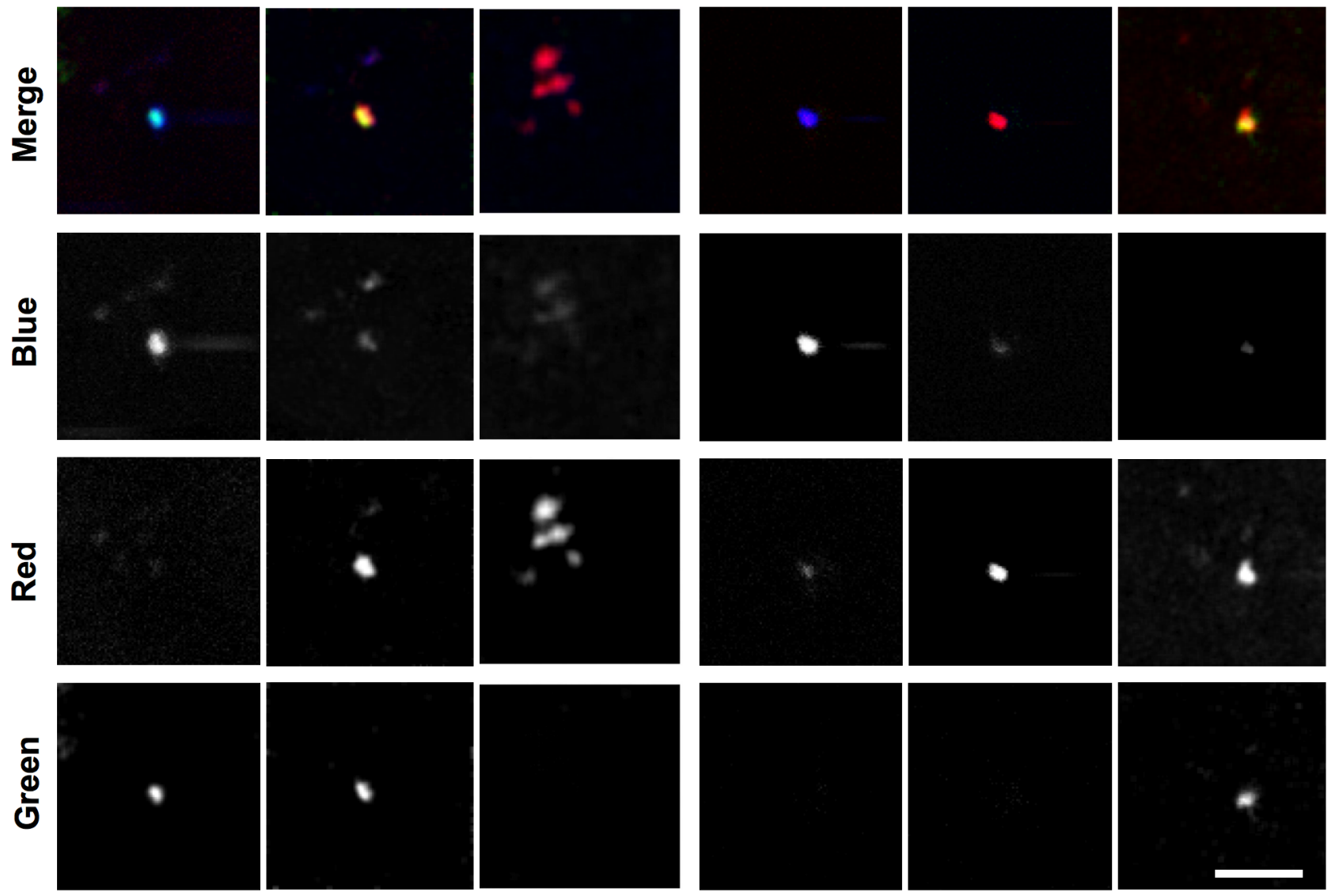

A

B

C

Loss of FoxP3-GFP

D

E

$F$

Gain of FoxP3-GFP
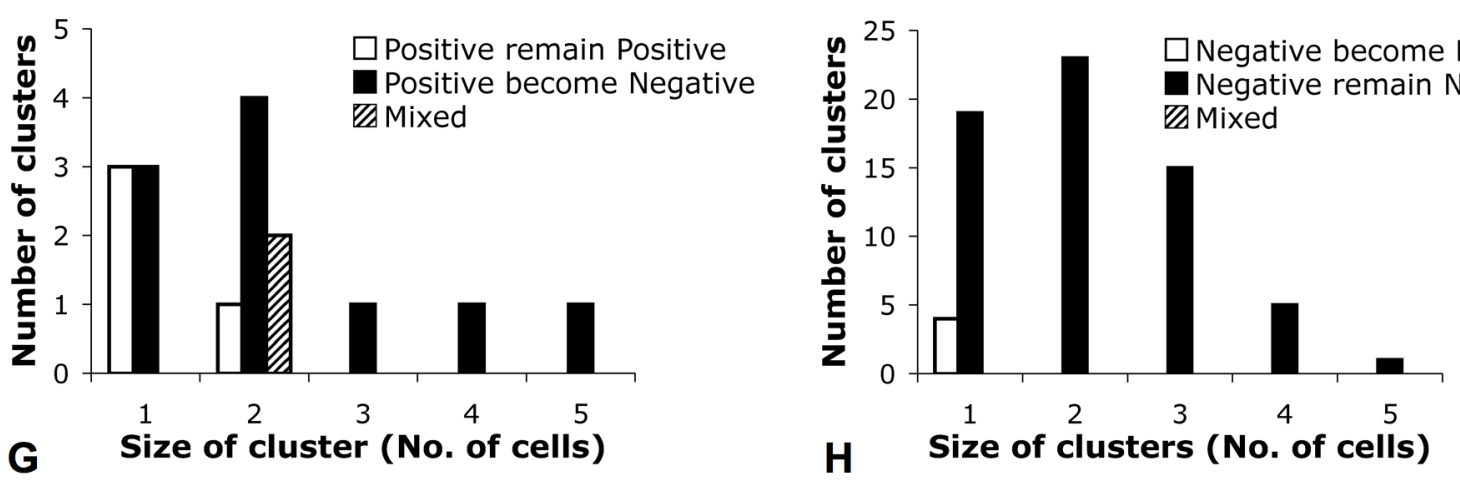

Figure 6. In vivo tracking of FoxP3-GFP switching on and off in $\mathrm{CD4}^{+} \mathbf{T}$ cells. $24 \mathrm{~h}$ after adoptive transfer of DiR-labeled FoxP3-GFP positive or negative T cells into RAG2 ${ }^{-1-}$ mice, T cells in skull BM were photoconverted and tracked longitudinally (blue: DiR, $>770 \mathrm{~nm}$; red: photoconvertedDiR, 670-720 nm; green: GFP, 509-547 nm). Series (A)-(C) shows that $48 \mathrm{~h}$ after photoconversion of DiR-labeled FoxP3-GFP-positive T cells (A: before photoconversion, blue+ red-green+; B: after photoconversion, blue- red+green+), some photoconverted cells (24/30) turned off expression of FoxP3-GFP (C: blue- red+ green-), indicating that non-Tregs can be generated from Tregs in the BM. Series (D)-(F) shows that $48 \mathrm{~h}$ after photoconversion of DiR-labeled FoxP3-GFP-negative T cells (D: before photoconversion, blue+ red-green-; E: after photoconversion, blue- red+ green-), a small portion of photoconverted cells (4/113) expressed FoxP3-GFP (F: blue - red+ green+), indicating that FoxP3-GFP Tregs can be generated from FoxP3-GFP-negative T cells in the BM. Scale bar: $50 \mu \mathrm{m}$. Charts $(\mathrm{G})$ and $(\mathrm{H})$ show the number of clusters of FoxP3-GFP positive or negative cells $48 \mathrm{~h}$ after photoconversion. 20 of 25 FoxP3-GFP-negative cells, which derived from 19 FoxP3-GFP-positive cells, made clusters. All 4 FoxP3-GFP-positive cells derived from 88 FoxP3-GFP-negative cells remained singlets.

doi:10.1371/journal.pone.0069257.g006

In vivo photoconversion of DiR-labeled T cells to track single cell fate

As a second example of the application of the dye-photoconversion method, we used an additional fluorescent reporter gene that marks cell differentiation or function, thus enabling us to track the in vivo differentiation status of individual cells over time. Here we use the technique to track CD4 T cell peripheral differentiation within the BM by examining the gain or loss of their FoxP3 
expression. FoxP3 is a transcription factor whose expression in a subset of CD4 T lymphocytes, called regulatory $\mathrm{T}$ cells (Tregs), confers immune suppressive function and plays a critical role in maintaining immune self-tolerance [43]. The development of FoxP3-GFP knock-in mice has provided a powerful means to identify the FoxP3-positive Treg cell population [42,44], but new questions have been raised about the stability of the FoxP3 expression after adoptive transfer [5-9]. In standard populationbased studies, it has been difficult to exclude the possibility that expansion of a small population of contaminating cells in the starting pool (for example, non-Tregs in a pool of supposedly purified Tregs or vise versa) could be misinterpreted as newly generated cells that lost/gained FoxP3 expression [5-8]. Moreover, these population-based studies do not provide information about the cellular location where these transitions take place. To overcome these limitations, we track the gain or loss of FoxP3GFP expression in GD4 T cells after highlighting them by the in vivo dye-photoconversion method. This method enabled unambiguous identification of whether the individual starting CD4 T cells were Tregs or non-Tregs, and provided information on the location where the switching of the phenotype took place in vivo.

After isolating FoxP3-positive Tregs from FoxP3-GFP mice, we adoptively transferred DiR-labeled Tregs into RAG2 $2^{-/-}$mice. $24 \mathrm{~h}$ later, the skull $\mathrm{BM}$ of the recipients were imaged, and individual T cells were photoconverted. $48 \mathrm{~h}$ after photoconversion, the skull BM was re-imaged to track FoxP3-GFP expression of the photoconverted cells. A total of 19 FoxP3-GFP-positive Tregs were photoconverted, and 48 h later, 32 photoconverted $\mathrm{T}$ cells were found in the skull BM cavities. Some photoconverted T cells formed clusters at the same location where a single $\mathrm{T}$ cell was previously photoconverted, indicating homeostatic proliferation of CD4 $\mathrm{T}$ cells in the BM of lymphopenic mice. Interestingly, 25 of the 32 photoconverted $\mathrm{T}$ cells did not express GFP $48 \mathrm{~h}$ after photoconversion (Figs. 6A-6C), indicating that some FoxP3positive Tregs turned off their FoxP3 expression. Additionally, 20 of 25 photoconverted FoxP3-GFP-negative $\mathrm{T}$ cells were found in clusters, suggesting that loss of FoxP3 expression in CD4 Tregs in the lymphopenic condition is associated with homeostatic proliferation (Fig. 6G).

We also performed the opposite experiment, starting with FoxP3-GFP-negative CD4 T cells isolated from the FoxP3-GFP mice. In recipients adoptively transferred with only DiR-labeled FoxP3-GFP-negative non-Tregs, 88 FoxP3-GFP-negative T cells were photoconverted, and $48 \mathrm{~h}$ later, 139 photoconverted T cells were found in the skull BM cavities, again indicating homeostatic proliferation. Four of the 139 photoconverted T cells expressed GFP, indicating that FoxP3-negative $\mathrm{T}$ cells can turn on FoxP3 expression in the BM (Figs. $6 \mathrm{D}-6 \mathrm{~F}$ and $6 \mathrm{H}$ ). These data directly demonstrate the instability of the FoxP3 transcription factor in CD4 $\mathrm{T}$ cells in the lymphopenic condition, indicating that Tregs and non-Tregs can switch their phenotypes in the BM.

\section{Discussion}

The DiR dye-photoconversion method uses a commercial lipophilic membrane dye and ratiometric imaging to distinguish photoconverted cells from non-photoconverted cells with high sensitivity. This robust method can be used to highlight and track individual cells to yield temporal and spatial information about the division, differentiation, and movement of cells at the single-cell level. Compared with photoswitchable [18-28] and photoconvertible [18,29-40] fluorescent proteins, the NIR photoconvertible membrane dye has a number of advantages: (i) Photoconversion of the $\mathrm{DiR}$ dye is permanent. Once photoconverted, the cell does not reacquire the original fluorescence signal. Additionally, the photoconverted fluorescence ratio is stable through cell division and is passed on to the daughter cells so that progeny of a photoconverted cell can be tracked over at least three cell divisions (Fig. 6C). In contrast, for photoconvertible fluorescent proteins, protein turnover leads to a loss of the photoconverted protein and gain of unconverted protein within $6 \mathrm{~h}$ of photoconversion [37] and the cell reverts back to the original unconverted color within $24 \mathrm{~h}$ of photoconversion in rapidly proliferating cells if no additional photoconversion is performed [41]. This color reversion is a critical obstacle to using photoconvertible fluorescent proteins to track cell division and differentiation. (ii) Any cells, including freshly isolated primary cells, can be labeled with DiR after a short incubation time with no known effect on their homing or proliferation [3]. The dye labeling method avoids gene transfection, which can affect cell differentiation or function. It also enables labeling of cells that cannot be maintained in culture (e.g., HSPCs) or transfected efficiently (e.g., T cells). Although dye transfer to adjacent cells can be a potential source of "environmental contamination" in cell cultures [46], we have not observed evidence of significant dye transfer in vivo. (iii) Finally, the third advantage is that the NIR dye can be used with GFP or other fluorescent reporters to track gene expression and cell differentiation.

The change in the fluorescence emission spectrum of the fluorophore used in the DiR dye, 1,1',3,3,3',3'-hexamethylindotrycarbocyanine iodide (HITC), has been noted previously by del Monte and Levy [47] and was attributed to chemical degradation. The change in emission after light activation may be due to an irreversible trans-cis photoisomerization of the DiR dye molecule, as described by Mishra, et al. [48]. We noted that the photoconversion does not occur when the dye is in solution, but only when it is bound to a cell, further suggesting that conformational change is key to the photoconversion.

Additionally, we noted that cells labeled with a similar dye, DiD $\left(\mathrm{DiIC}_{18}(5)\right.$ 'Vibrant DiD'; Invitrogen, Carlsbad, CA), at a concentration of $5 \mu \mathrm{M}$ for $15 \mathrm{~min}$ could also be photoconverted in vitro using $632 \mathrm{~nm}$ illumination, changing the detected fluorescence from approximately $670 \mathrm{~nm}$ to something near $570 \mathrm{~nm}$. Although the illumination wavelength and intensity were not ideal for using the photoconversion technique in vivo with the DiD dye, it suggests that it may be possible to photoconvert the other members of the $\mathrm{DiIG}_{18}$ family in addition to DiR.

Using the dye-photoconversion method in combination with IVM, we have shown that HSPCs injected into irradiated recipients divide at the location on the endosteal surface where they initially home within one hour after intravenous injection (Fig. 5). While previous studies have shown that the HSPC microenvironment, or niche, plays an important role in regulating stem cell division and differentiation, those studies did not directly examine the precise location where these events occurred. Previous studies have identified two HSPC niches, one residing near the endosteum $[1,4]$ and the other separate from osteoblasts and near BM sinusoidal endothelium away from the endosteum (the vascular niche) [2]. It has been suggested that the endosteal niche may favor stem cell quiescence while the vascular niche may favor proliferation, and the HSPCs may shuttle back and forth between the two niches [49]. We have recently shown that the endosteal niche is in fact perivascular [3], so the two niches may have overlapping perivascular components. Here we have further provided evidence that HSPGs do not have to migrate away from the location where they initially home after transplantation in order to proliferate. With additional cell lineage markers, it will be possible to examine if there are specific locations in the BM that 
support HSPC differentation. It is possible that instead of two separate but static niches, a single niche as a dynamic entity can support HSPC transition through multiple cell states.

Using the same method, we have also shown that homeostatic proliferation of $\mathrm{CD}^{+} \mathrm{T}$ cells occurs in the BM (Fig. 6). When combined with a fluorescent reporter gene (FoxP3-GFP), we have shown that $\mathrm{CD}^{+} \mathrm{T}$ cells can both acquire and lose FoxP3 expression in the BM after adoptive transfer to lymphopenic mice (Fig. 6). Because we tracked individual cells with a well-defined phenotype, this method avoids the problem of heterogeneity in the starting cell pool that has complicated the interpretation of previous population-based studies [3,5-9]. Additionally, our method allows direct identification of the cellular location where these transitions take place. The finding that Treg cells can lose FoxP3 expression during homeostatic proliferation has been suggested previously [5,50,51]. Further studies are needed to determine if this loss is a transient phenomenon and whether the Treg cells will reacquire FoxP3 expression at a later stage, which seems possible given the acquired GFP expression in a subset of GFP-negative CD4-positive transplanted lymphocytes.

\section{References}

1. Calvi LM, Adams GB, Weibrecht KW, Weber JM, Olson DP, et al. (2003) Osteoblastic cells regulate the haematopoietic stem cell niche. Nature 425: 841846.

2. Kiel MJ, Yilmaz OH, Iwashita T, Terhorst C, Morrison SJ (2005) SLAM family receptors distinguish hematopoietic stem and progenitor cells and reveal endothelial niches for stem cells. Cell 121: 1109-1121.

3. Lo Celso C, Fleming HE, Wu JW, Zhao CX, Miake-Lye S, et al. (2009) Liveanimal tracking of individual haematopoietic stem/progenitor cells in their niche. Nature 457: 92-96.

4. Zhang J, Niu C, Ye L, Huang H, He X, et al. (2003) Identification of the haematopoietic stem cell niche and control of the niche size. Nature 425: 836841.

5. Komatsu N, Mariotti-Ferrandiz ME, Wang Y, Malissen B, Waldmann H, et al. (2009) Heterogeneity of natural Foxp3+ T cells: a committed regulatory T-cell lineage and an uncommitted minor population retaining plasticity. Proc Natl Acad Sci USA 106: 1903-1908.

6. Liang S, Alard P, Zhao Y, Parnell S, Clark SL, et al. (2005) Conversion of CD4+ CD25- cells into CD4+ CD25+ regulatory $\mathrm{T}$ cells in vivo requires $\mathrm{B} 7$ costimulation, but not the thymus. J Exp Med 201: 127-137.

7. Sharma MD, Hou DY, Liu Y, Koni PA, Metz R, et al. (2009) Indoleamine 2,3dioxygenase controls conversion of Foxp3+ Tregs to TH17-like cells in tumordraining lymph nodes. Blood 113: 6102-6111.

8. Wang L, Pino-Lagos K, de Vries VC, Guleria I, Sayegh MH, et al. (2008) Programmed death 1 ligand signaling regulates the generation of adaptive Foxp3+CD4+ regulatory T cells. Proc Natl Acad Sci USA 105: 9331-9336.

9. Zhou L, Chong MMW, Littman DR (2009) Plasticity of CD4+ T cell lineage differentiation. Immunity 30: 646-655.

10. Eilken HM, Nishikawa S-I, Schroeder T (2009) Continuous single-cell imaging of blood generation from haemogenic endothelium. Nature 457: 896-900.

11. Wu M, Kwon HY, Rattis F, Blum J, Zhao G, et al. (2007) Imaging hematopoietic precursor division in real time. Cell Stem Cell 1: 541-554.

12. Cahalan MD, Parker I, Wei SH, Miller MJ (2002) Two-photon tissue imaging: seeing the immune system in a fresh light. Nat Rev Immunol 2: 872-880.

13. Germain RN, Bajénoff M, Castellino F, Chieppa M, Egen JG, et al. (2008) Making friends in out-of-the-way places: how cells of the immune system get together and how they conduct their business as revealed by intravital imaging. Immunol Rev 221: 163-181.

14. Mempel TR, Pittet MJ, Khazaie K, Weninger W, Weissleder R, et al. (2006) Regulatory $\mathrm{T}$ cells reversibly suppress cytotoxic $\mathrm{T}$ cell function independent of effector differentiation. Immunity 25: 129-141.

15. Miller MJ, Wei SH, Parker I, Cahalan MD (2002) Two-photon imaging of lymphocyte motility and antigen response in intact lymph node. Science 296: 1869-1873.

16. Pittet MJ, Mempel TR (2008) Regulation of T-cell migration and effector functions: insights from in vivo imaging studies. Immunol Rev 221: 107-129.

17. Fan Z, Spencer JA, Lu Y, Pitsillides CM, Singh G, et al. (2010) In vivo tracking of 'color-coded' effector, natural and induced regulatory T cells in the allograft response. Nat Med 16: 718-722.

18. Adam V, Lelimousin M, Boehme S, Desfonds G, Nienhaus K, et al, (2008) Structural characterization of IrisFP, an optical highlighter undergoing multiple photo-induced transformations. Proc Natl Acad Sci USA 105: 18343-18348.

19. Ando R, Mizuno H, Miyawaki A (2004) Regulated fast nucleocytoplasmic shuttling observed by reversible protein highlighting. Science 306: 1370-1373.
In summary, we have developed a simple in vivo cell tracking method using DiR dye-photoconversion that can be used with any cell type that can be labeled ex vivo and adoptively transferred into recipient animals. Labeling is brief and does not require cell transfection. The NIR-wavelength spectral range of the DiR dye leaves the entire visible spectrum open for use with other fluorescent protein-based reporters that will enable multi-lineage tracking with single-cell resolution longitudinally over time in live animals.

\section{Acknowledgments}

We wish to thank Dr. Daniel Côté for providing the image capture software, iPhoton.

\section{Author Contributions}

Conceived and designed the experiments: CPL ALC JF. Performed the experiments: ALG JF JWW CL JMR. Analyzed the data: ALC JF JWW RT. Wrote the paper: ALC JF CPL. Provided expertise in HSPC biology: DTS. Provided expertise in T cell biology: TBS. Conceived dyephotoconversion strategy: CPL.

20. Ando R, Flors C, Mizuno H, Hofkens J, Miyawaki A (2007) Highlighted generation of fluorescence signals using simultaneous two-color irradiation on Dronpa mutants. Biophys J 92: L97-99.

21. Andresen M, Wahl MC, Stiel AC, Gräter F, Schäfer LV, et al. (2005) Structure and mechanism of the reversible photoswitch of a fluorescent protein. Proc Natl Acad Sci USA 102: 13070-13074.

22. Andresen M, Stiel AC, Fölling J, Wenzel D, Schönle A, et al. (2008) Photoswitchable fluorescent proteins enable monochromatic multilabel imaging and dual color fluorescence nanoscopy. Nat Biotechnol 26: 1035-1040.

23. Chirico G, Cannone F, Diaspro A, Bologna S, Pellegrini V, et al. (2004) Multiphoton switching dynamics of single green fluorescent proteins. Phys Rev E Stat Nonlin Soft Matter Phys 70: 030901.

24. Hofmann M, Eggeling C, Jakobs S, Hell SW (2005) Breaking the diffraction barrier in fluorescence microscopy at low light intensities by using reversibly photoswitchable proteins. Proc Natl Acad Sci USA 102: 17565-17569.

25. Patterson GH, Lippincott-Schwartz J (2002) A photoactivatable GFP for selective photolabeling of proteins and cells. Science 297: 1873-1877.

26. Stiel AC, Trowitzsch S, Weber G, Andresen M, Eggeling C, et al. (2007) 1.8 A bright-state structure of the reversibly switchable fluorescent protein Dronpa guides the generation of fast switching variants. Biochem J 402: 35-42.

27. Stiel AC, Andresen M, Bock H, Hilbert M, Schilde J, et al. (2008) Generation of monomeric reversibly switchable red fluorescent proteins for far-field fluorescence nanoscopy. Biophys J 95: 2989-2997.

28. Verkhusha VV, Sorkin A (2005) Conversion of the monomeric red fluorescent protein into a photoactivatable probe. Chem Biol 12: 279-285.

29. Ando R, Hama H, Yamamoto-Hino M, Mizuno H, Miyawaki A (2002) An optical marker based on the UV-induced green-to-red photoconversion of a fluorescent protein. Proc Natl Acad Sci USA 99: 12651-12656.

30. Chudakov DM, Verkhusha VV, Staroverov DB, Souslova EA, Lukyanov S, et al. (2004) Photoswitchable cyan fluorescent protein for protein tracking. Nat Biotechnol 22: 1435-1439.

31. Gurskaya NG, Verkhusha VV, Shcheglov AS, Staroverov DB, Chepurnykh TV, et al. (2006) Engineering of a monomeric green-to-red photoactivatable fluorescent protein induced by blue light. Nat Biotechnol 24: 461-465.

32. Habuchi S, Tsutsui H, Kochaniak AB, Mivawaki A, Van Oijen AM (2008) mKikGR, a monomeric photoswitchable fluorescent protein. PLoS ONE 3: e3944.

33. Kremers G-J, Hazelwood KL, Murphy CS, Davidson MW, Piston DW (2009) Photoconversion in orange and red fluorescent proteins. Nat Meth 6: 355-358.

34. Marchant JS, Stutzmann GE, Leissring MA, LaFerla FM, Parker I (2001) Multiphoton-evoked color change of DsRed as an optical highlighter for cellular and subcellular labeling. Nat Biotechnol 19: 645-649.

35. McKinney SA, Murphy CS, Hazelwood KL, Davidson MW, Looger LL (2009) A bright and photostable photoconvertible fluorescent protein. Nat Meth 6: $131-133$.

36. Nowotschin S, Hadjantonakis A-K (2009) Use of KikGR a photoconvertible green-to-red fluorescent protein for cell labeling and lineage analysis in ES cells and mouse embryos. BMC Dev Biol 9: 49

37. Tomura M, Yoshida N, Tanaka J, Karasawa S, Miwa Y, et al. (2008) Monitoring cellular movement in vivo with photoconvertible fluorescence protein "Kaede" transgenic mice. Proc Natl Acad Sci USA 105: 10871-10876.

38. Tsutsui H, Karasawa S, Shimizu H, Nukina N, Miyawaki A (2005) Semirational engineering of a coral fluorescent protein into an efficient highlighter. EMBO Rep 6: 233-238. 
39. Wiedenmann J, Ivanchenko S, Oswald F, Schmitt F, Röcker C, et al. (2004) EosFP, a fluorescent marker protein with UV-inducible green-to-red fluorescence conversion. Proc Natl Acad Sci USA 101: 15905-15910.

40. Zhang M, Chang H, Zhang Y, Yu J, Wu L, et al. (2012) Rational design of true monomeric and bright photoactivatable fluorescent proteins. Nat Meth 9: 727729.

41. Griswold SL, Sajja KC, Jang G-W, Behringer RR (2011) Generation and characterization of iUBC-KikGR photoconvertible transgenic mice for live time lapse imaging during development. Genesis Ahead of print, accepted Jan 11, 2011.

42. Fontenot JD, Gavin MA, Rudensky AY (2003) Foxp3 programs the development and function of CD4+CD25+ regulatory T cells. Nat Immunol 4: 330-336.

43. Tang Q, Bluestone JA (2008) The Foxp3+ regulatory T cell: a jack of all trades, master of regulation. Nat Immunol 9: 239-244.

44. Wan YY, Flavell RA (2005) Identifying Foxp3-expressing suppressor T cells with a bicistronic reporter. Proc Natl Acad Sci USA 102: 5126-5131.
45. Veilleux I, Spencer JA, Biss DP, Cote D, Lin C (2008) In vivo Cell Tracking with Video Rate Multimodality Laser Scanning Microscopy. JSTQE Biophotonics 14: 10-18.

46. Lassailly F, Griessinger E, Bonnet D (2010) "Microenvironmental contaminations" induced by fluorescent lipophilic dyes used for noninvasive in vitro and in vivo cell tracking. Blood 115: 5347-5354.

47. del Monte F, Levy D (1995) Near-infrared dyes encapsulated in sol-gel matrices. Chem Mater 7: 292-298.

48. Mishra A, Behera RK, Behera PK, Mishra BK, Behera GB (2000) Cyanines during the 1990s: A Review. Chem Rev 100: 1973-2012.

49. Li L, Clevers H (2010) Coexistence of quiescent and active adult stem cells in mammals. Science 327: 542-545.

50. Strom TB, Koulmanda M (2009) Recently discovered T cell subsets cannot keep their commitments. J Am Soc Nephrol 20: 1677-1680.

51. Zhou X, Bailey-Bucktrout SL, Jeker LT, Penaranda C, Martínez-Llordella M, et al. (2009) Instability of the transcription factor Foxp3 leads to the generation of pathogenic memory T cells in vivo. Nat Immunol 10: 1000-1007. 